\title{
Shear Behavior of AA6061 Aluminum in the Semisolid State Under Isothermal and Nonisothermal Conditions
}

\author{
E. GIRAUD, M. SUERY, and M. CORET
}

The shear behavior of a 6061 aluminum alloy was studied in the semisolid state at large solid fractions. The tests were carried out either at constant temperature after partial solidification (i.e., isothermal shear tests) or during solidification at low cooling rate (i.e., nonisothermal shear tests). In isothermal conditions, results show that (1) the mechanical behavior depends on the volume fraction of the solid phase present in the sample at the temperature of the test, (2) there is a critical solid fraction corresponding to the coalescence of the solid grains beyond which shear stress increases very sharply with increasing solid fraction, and (3) the mushy alloy exhibits viscoplastic behavior with a strain-rate-sensitivity parameter close to about 0.17 . In nonisothermal conditions, results show that stress increases continuously with decreasing temperature whatever the strain rate. However, at high strain rate, it was observed that cracks developed when the solid fraction approaches 1, leading to a slower stress increase compared to that observed at low strain rate. Finally, modeling of this behavior is carried out by considering a cohesion parameter of the solid phase, which depends on solid fraction and strain rate.

\section{INTRODUCTION}

SOLIDIFICATION of metallic alloys involves the transformation of the liquid phase into one or more solid phases over a given temperature interval. During this transformation, several transitions are usually defined separating various types of behavior. ${ }^{[1-3]}$ The first transition corresponds to the coherency solid fraction, which separates the domain where the solid grains are free to move in the liquid from the domain where they begin to mechanically interact. Below the coherency solid fraction, the viscosity of the material is close to that of the liquid. Just above this solid fraction, the material is able to transmit shear strains, but the bonds between the solid grains are too weak so that the material cannot transmit tensile strains. In the case of $\mathrm{Al}$ alloys, this transition occurs for solid fractions lower than 0.6. ${ }^{[1,2,4]}$ The second transition separates the domain where the liquid is able to flow between the solid grains from the domain where intergranular flow is no longer possible. At this point, the liquid is present only as films between the solid grains. The material can then transmit shear and tensile strains. In general, this second transition starts at a solid fraction of about $0.9 .^{[1,2]}$ Finally, the coalescence solid fraction corresponds to the point where the solid starts to coalesce and

E. GIRAUD, formerly with the Universite de Grenoble, is now Postdoctoral Researcher with the MMS, University of Liège, 4000 Liège, Belgium. Contact e-mail: eliane.giraud@ulg.ac.be M. SUERY, Research Director, is with the Universite de Grenoble, SIMaP, UMR CNRS 5266, 38402 Saint Martin d'Hères Cedex, France. M. CORET, Associate Professor, is with the Universite de Lyon, LaMCoS, INSALyon, UMR CNRS 5514, 69621 Villeurbanne, France. form a continuous skeleton with a mechanical strength close to that of the solid. Several investigations ${ }^{[1,2,4-7]}$ showed that this transition occurs for solid fractions close to 0.97 in the case of $\mathrm{Al}$ alloys.

The solid fraction for which liquid flow can no longer be possible is very critical during casting and fusion welding processes, since it corresponds to the possible occurrence of hot tearing. ${ }^{[1,6,8-10]}$ Indeed, at this stage, strains generated in the material by solidification shrinkage, thermal contraction, and external loading cannot be accommodated by liquid flow, thus leading to crack formation. ${ }^{[1]}$ The study of the hot tearing phenomenon, therefore, requires investigation of the behavior of the alloy in the range 0.90 to 0.97 , where the alloy is relatively brittle. ${ }^{[1,5,6,8,9,12]}$

Modeling of this phenomenon requires, on the one hand, the knowledge of the rheological behavior of the alloy in this solid fraction range and, on the other hand, a criterion for the formation of cracks. Since cracks are usually the result of tensile strains, the tensile behavior of the alloy close to the end of solidification is of prime importance. This behavior was investigated recently in the case of various $\mathrm{Al}$ alloys, ${ }^{[5-7,9,11-15]}$ and it has been shown, in particular, that this behavior strongly depends on the thermal history experienced by the alloy. ${ }^{[5,6]}$ It is indeed important to consider the behavior during partial solidification and not during partial melting, because cracks form during cooling of the alloy from the liquid state.

Although the tensile behavior is responsible for hot tear formation, other stress states can be generated in the solidifying part depending on geometry and boundary conditions. ${ }^{[14]}$ Thus, in order to model the hot cracking phenomenon during a welding process, it is necessary to investigate the behavior of the solidifying alloy for any stress state. 
The aim of this article is to report experimental results concerning the shear behavior of a 6061 alloy during solidification both in isothermal and nonisothermal conditions. Isothermal conditions allow investigation of the behavior of the mushy state for various solid fractions, whereas nonisothermal tests are closer to the conditions encountered during hot crack formation. Since this work is carried out in the context of hot tearing, the study was limited to large solid fractions greater than $0.8^{[1,10]}$ The same type of study was previously carried out to determine the tensile behavior of a 6061 alloy in its semisolid state and has been reported in References 5 and 16.

\section{EXPERIMENTAL PROCEDURE}

\section{A. Material}

The AA6061 alloy studied in this work is the same as that used in the tensile test experiments reported previously. ${ }^{[5,16]}$ It was supplied by ALMET as rolled plates, $50 \mathrm{~mm}$ in thickness and in the T6 condition (solution heat treated and then artificially aged). The composition is given in Table I. The initial microstructure is not shown since the material is entirely melted before the shear tests start.

\section{B. Shear Tests}

A schematic view of the shear test apparatus is shown in Figure 1. It is basically similar to the one used by Ludwig et al. in a previous work on $\mathrm{Al}-\mathrm{Cu}$ alloys. ${ }^{[7,17,18]}$ It consists of two coaxial cylinders, which are able to exhibit a translatory movement one with respect to the other. Both are threaded to avoid slippage of the alloy while being sheared and are coated with AQUAGEL $87^{*}$ to prevent attack from

*AQUAGEL 87 is a trademark of Acheson Colloids Company, Port Huron, MI.

liquid aluminum. The outer cylinder is connected to the frame of a tension machine, and the inner cylinder is connected to the crosshead of the machine by using steel bars.

Thermocouples ( $\mathrm{K}$ type of 1-mm diameter) are inserted in the wall and in the bottom of the outer cylinder to monitor the temperature. The solid alloy is placed initially in the outer cylinder with the inner cylinder above it. An additional thermocouple is put in the sample to check the temperature. Ludwig et al. checked the temperature gradient in the sample in the vertical direction and found that it is less than $1.3 \mathrm{~K} / \mathrm{cm} \cdot{ }^{[17]}$

The alloy is melted and the downward movement of the inner cylinder allows the alloy to fill the gap between

Table I. Composition of the Alloy (Weight Percent)

\begin{tabular}{ccccccc}
\hline $\mathrm{Mg}$ & $\mathrm{Si}$ & $\mathrm{Cu}$ & $\mathrm{Fe}$ & $\mathrm{Cr}$ & $\mathrm{Mn}$ & $\mathrm{Al}$ \\
\hline 0.93 & 0.61 & 0.28 & 0.26 & 0.2 & 0.12 & balance \\
\hline
\end{tabular}

the cylinders. The alloy is then partially solidified at a controlled constant cooling rate, equal to $0.3 \mathrm{~K} / \mathrm{s}$. The cooling rate is quite small in these experiments owing to the relatively large mass of the cylinders, which does not allow cooling of the alloy at a larger constant cooling rate. During cooling, thermal strains were accommodated so as to avoid any deformation of the specimen. At a given temperature, a constant velocity is imposed to the inner cylinder, thus shearing the alloy at a constant rate. To avoid depression during the motion of the inner cylinder, fiber insulation is put between the wall and the bottom of the outer cylinder, which allows air entering under the inner cylinder during shearing without leakage of liquid.

Two types of tests were performed: isothermal tests and nonisothermal ones during which the temperature is decreased at a rate of $0.3 \mathrm{~K} / \mathrm{s}$ from a given temperature (i.e., a given solid fraction present in the alloy). Figure 2 shows the thermal cycle imposed on the alloy during both tests.

The temperature was converted into solid fraction by using a calculation based on the ProPhase software (developed by Alcan CRV, Voreppe, France). Figure 3 shows the variation of solid fraction with temperature.

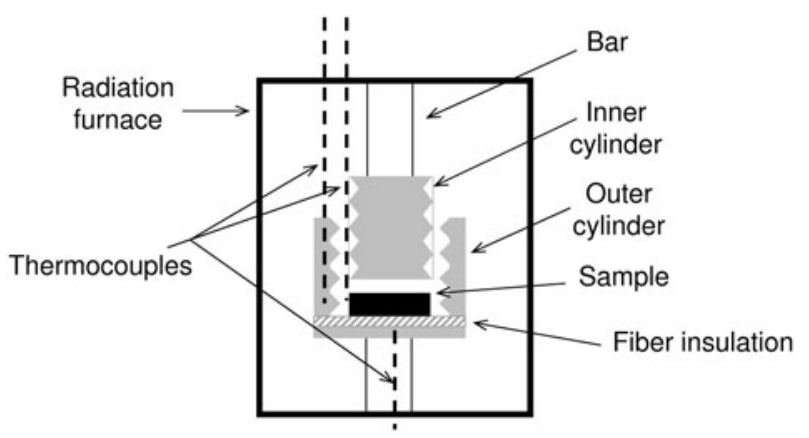

Fig. 1-Sketch of the experimental device.

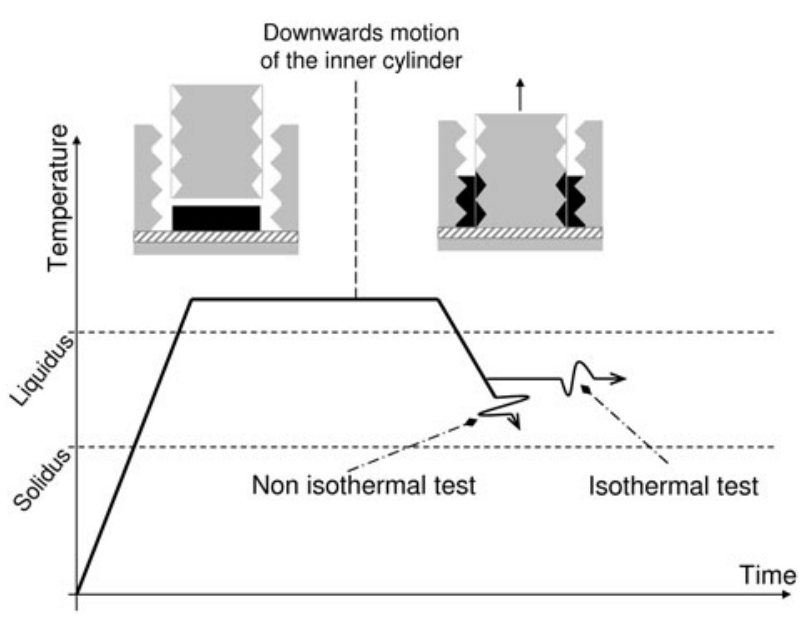

Fig. 2-Thermal cycle imposed to the alloy during isothermal and nonisothermal experiments. 


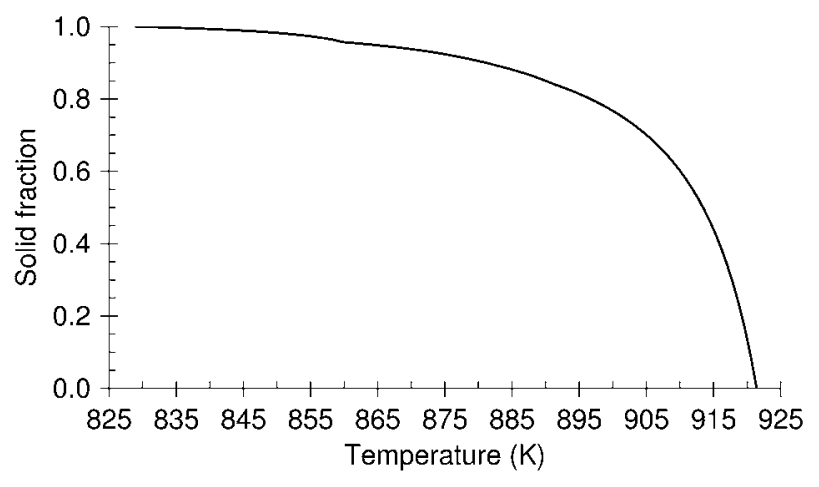

Fig. 3-Variation of solid fraction with temperature according to ProPhase calculation for a cooling rate of $0.3 \mathrm{~K} / \mathrm{s}$.

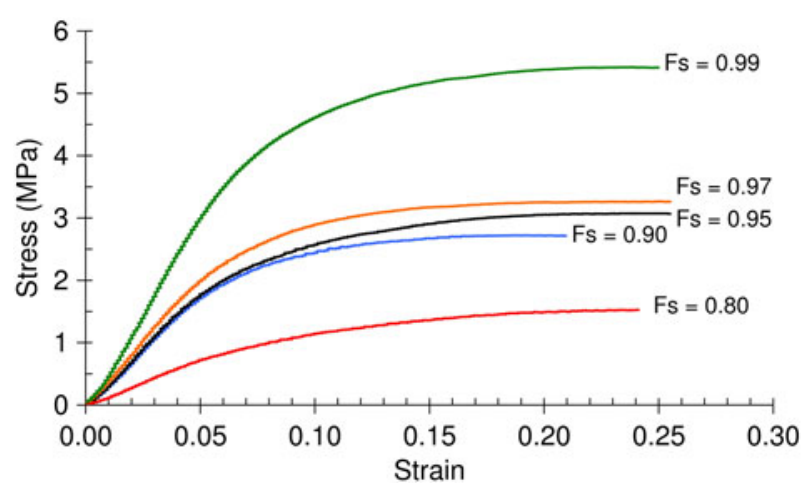

Fig. $4-$ Von Mises stress as a function of strain obtained during isothermal shear tests carried out at various solid fractions $\left(F_{\mathrm{s}}\right)$ at a displacement rate of $0.006 \mathrm{~mm} / \mathrm{s}$ (i.e., a strain rate of $0.001 \mathrm{~s}^{-1}$ ).

\section{EXPERIMENTAL RESULTS}

\section{A. Shear Behavior of the Alloy Under Isothermal Conditions in the Mushy State}

Isothermal shear tests allow the effects of strain, strain rate, and solid fraction to be investigated in a decoupled manner. Figure 4 shows the variation of the Von Mises stress as a function of the equivalent strain $\varepsilon_{e}$ for shear tests carried out at a displacement rate of $6 \cdot 10^{-3} \mathrm{~mm} / \mathrm{s}$ at various temperatures corresponding to various solid fractions.

For this plot, the Von Mises stress is defined as $\sqrt{3} \cdot \tau$, where $\tau$ is the shear stress applied to the alloy and is equal to $F / 2 \cdot \pi \cdot R_{i} \cdot h$, with $F$ the measured force, $R_{i}$ the radius of the inner cylinder $(18 \mathrm{~mm})$, and $h$ the height of the alloy in the outer cylinder (approximately $25 \mathrm{~mm}$ ). The equivalent strain $\varepsilon_{e}$ is equal to $\gamma / \sqrt{3}$, where $\gamma$ is the shear strain defined as the displacement of the inner cylinder divided by the gap between the two cylinders $(3.25 \mathrm{~mm})$. The displacement rate is converted into an equivalent strain rate $\dot{\varepsilon}_{\mathrm{e}}$ according to $\dot{\varepsilon}_{\mathrm{e}}=\dot{\gamma} / \sqrt{3}$, where $\dot{\gamma}$ is defined as the displacement rate divided by the gap between the two cylinders.

It should be noted that the reproducibility of the experiments is very good since the uncertainty on the stress value is less than about $0.2 \mathrm{MPa}$. As expected,

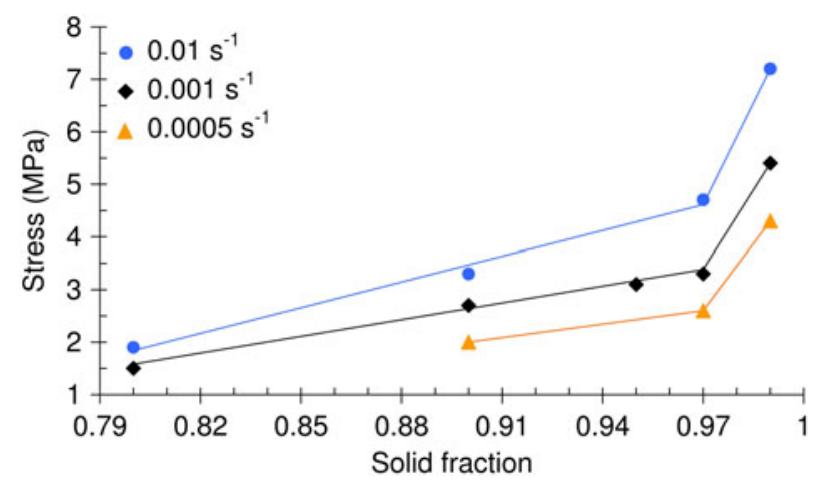

Fig. 5-Plateau stress as a function of solid fraction for isothermal shear tests performed at three strain rates: 0.01, 0.001, and $0.0005 \mathrm{~s}^{-1}$.

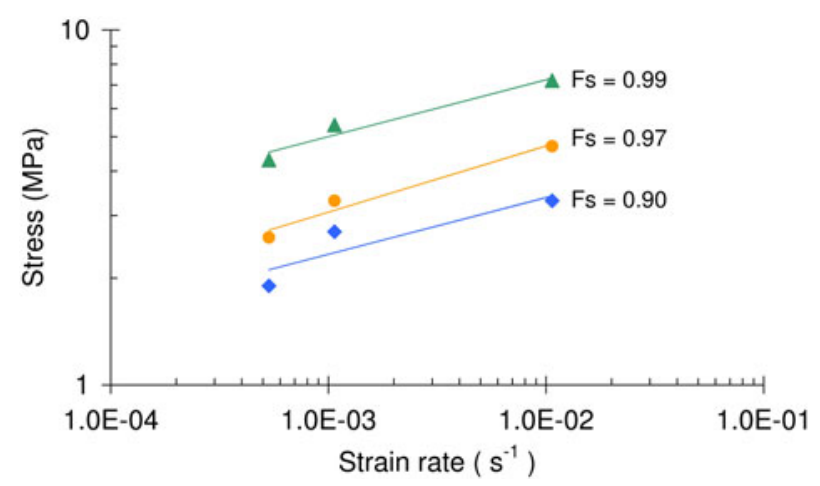

Fig. 6-Plateau stress-strain rate curves in a logarithmic scale for various solid fractions $\left(F_{\mathrm{s}}\right)$ and under isothermal conditions.

stress increases with increasing solid fraction, and whatever the solid fraction, the plateau stress is reached after a strain of about 0.15 to 0.20 .

Taking the value of the plateau stress for each solid fraction allows plotting the variation of the plateau stress as a function of the solid fraction (Figure 5). Since tests were carried out at various strain rates, Figure 5 shows also the results for these strain rates. It is clear from the figure that stress increases relatively slowly for solid fractions smaller than 0.97 and then more rapidly beyond 0.97 .

Stress increases also with increasing strain rate, indicating that the alloy exhibits a viscoplastic behavior. Figure 6 shows a plot of stress $v s$ equivalent strain rate on a log-log scale for various solid fractions. The straight lines indicate that a power-law equation applies with nearly constant values of the strain-rate-sensitivity parameter for the various solid fractions. It is approximately equal to 0.17 , leading to a stress exponent equal to about 6 .

The microstructures of the sheared specimens at a strain rate of $0.001 \mathrm{~s}^{-1}$ are shown in Figure 7 for specimens deformed up to about 0.25 equivalent strain at two different solid fractions, 0.90 and 0.97 . Obviously, these observations were carried out after complete solidification of the specimen and cooling to room temperature. The microstructures look very 

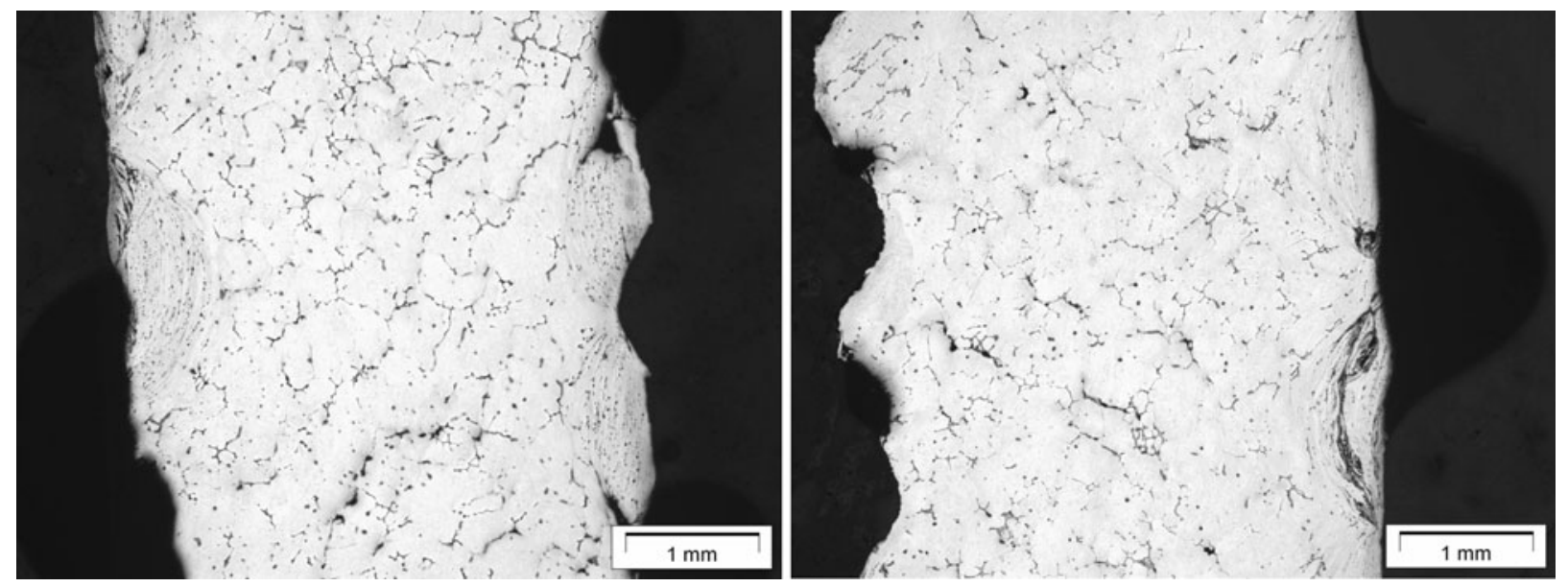

Fig. 7-Microstructures obtained after isothermal shear experiments at a strain rate of $0.001 \mathrm{~s}^{-1}$ and at a solid fraction of (a) 0.9 and (b) 0.97-Flick's etch.

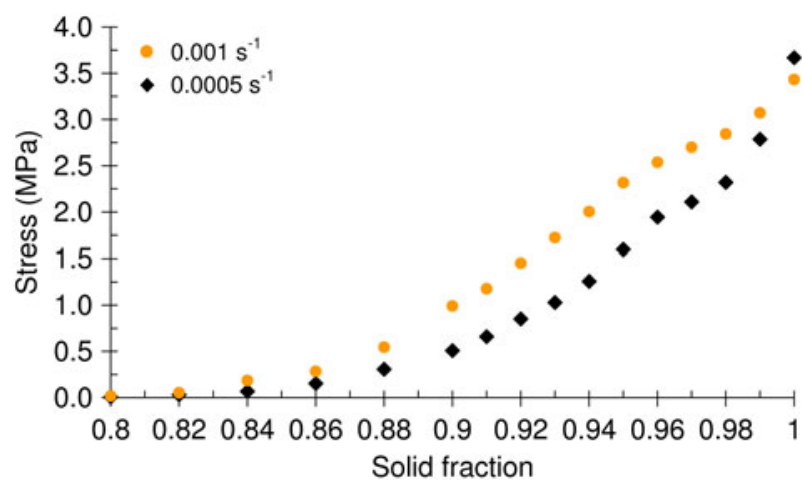

Fig. 8-Variation of the Von Mises stress with solid fraction during nonisothermal shear tests carried out with a cooling rate of $0.3 \mathrm{~K} / \mathrm{s}$, an initial solid fraction of 0.8 , and a strain rate of 0.001 or $0.0005 \mathrm{~s}^{-1}$.

similar with no evidence of damage induced by deformation. There is also no sign of preferential orientation of the grains.

\section{B. Shear Behavior of the Mushy State Under Nonisothermal Conditions}

By using the same apparatus as for the isothermal tests, shear tests were carried out during solidification of the alloy, starting the experiment at a solid fraction equal to 0.8 . As stated previously, the cooling rate was set at $0.3 \mathrm{~K} / \mathrm{s}$. Figure 8 shows the variation of the Von Mises stress with solid fraction for two tests carried out at $5 \cdot 10^{-4}$ and $1 \cdot 10^{-3} \mathrm{~s}^{-1}$. Stress increases obviously with increasing solid fraction, but the increase follows different kinetics depending on the solid fraction range. It increases initially slowly up to a solid fraction of 0.9 and then more rapidly. At a solid fraction of 0.96 , the stress increase slows again leading to stress values of about $3.5 \mathrm{MPa}$ at a solid fraction equal to 1 . For this solid fraction, the stress for the lower strain rate becomes larger than that for the higher strain rate. The slowdown of the stress increase at a solid fraction of 0.96 is surprising; we rather expect an abrupt increase since this solid fraction corresponds to the coalescence solid fraction. This result will be discussed in Section IV-B.

\section{DISCUSSION}

\section{A. Behavior Under Isothermal Conditions}

When shear is performed under isothermal conditions, Figure 4 shows that stress increases relatively slowly with increasing strain and reaches a steady state after about 20 pct. This behavior is very different from that observed for a fully solid alloy for which the steady state is obtained much more rapidly. This slow increase is correlated with the rearrangement of the dendrites, which can occur owing to the presence of interdendritic liquid. ${ }^{[14,19,20]}$ This rearrangement is obviously possible at large liquid fraction, but the figure shows that it still occurs when the solid fraction is as high as 0.99 . A similar behavior was previously observed in the case of other aluminum alloys. ${ }^{[7]}$

Stress increases with increasing solid fraction, as shown in Figure 5. It increases slowly up to a solid fraction equal to 0.97 and then much more rapidly. This solid fraction corresponds to the coalescence solid fraction of the dendrites, as already observed during tensile testing. ${ }^{[4,5,16]}$ For this solid fraction, solid bridges are becoming predominant so that deformation involves important deformation of the solid skeleton. However, this deformation does not induce damage of the material, as shown in Figure 7. This figure shows in addition that the microstructure of the alloy after cooling to room temperature does not depend on the solid fraction at the beginning of the shear test. Therefore, it can be concluded that observations after complete solidification are not appropriate to reveal the microstructure present in the mushy state. Indeed, cooling from the temperature of the test to room temperature was too slow to freeze the microstructure and to allow distinguishing the solid present when the alloy was semisolid from that formed upon cooling. 
Even at lower solid fraction, solid deformation should occur since the behavior of the alloy is viscoplastic. Indeed, Figure 6 shows that the strain-rate-sensitivity parameter ranges between 0.16 and 0.19 for solid fractions in the range 0.9 to 1 . These values can be considered as characteristic of the fully solid phase behavior. However, there appears to be a slope change at a shear rate of $10^{-3} \mathrm{~s}^{-1}$ with slightly lower stresses at a shear rate of $5 \cdot 10^{-4} \mathrm{~s}^{-1}$. Since this shear rate corresponds to long deformation times compared to higher shear rates, it is possible that some evolution of the microstructure occurred during the test leading to a slight decrease of the shear stress compared to that measured without any evolution.

This change in the mechanism of deformation of the mushy alloy at a given solid fraction (here, 0.97), which is identical whatever the stress state (tensile or shear), is in agreement with the results obtained by Dahle et al. on Al-Cu alloys. ${ }^{[14]}$ They have shown that the deformation of a semisolid material splits up into two groups of mechanisms: one group related to grain rearrangement and the other one related to the grain deformation and breaking of solid-solid contacts. Around the coherency, the mechanism of deformation corresponds to the first one. At very high solid fractions, the behavior is dominated by the deformation of the grains themselves. For intermediate solid fractions, the two types of mechanism are occurring. Thus, when the dendritic network is established, deformation occurs by deformation and fracture of dendrites, independent of whether the deformation occurs in shear or tension. The strength of the mushy alloy, independent of deformation mode, is therefore a result of the total area of solid joints that must be broken and the strength of these joints, which leads to a convergence of deformation mechanisms in tension and shear at high solid fractions. In other words, in the conditions for which hot cracking phenomenon occurs (i.e., at high solid fractions), tensile or shear experiments lead to the same behavior of the semisolid alloy.
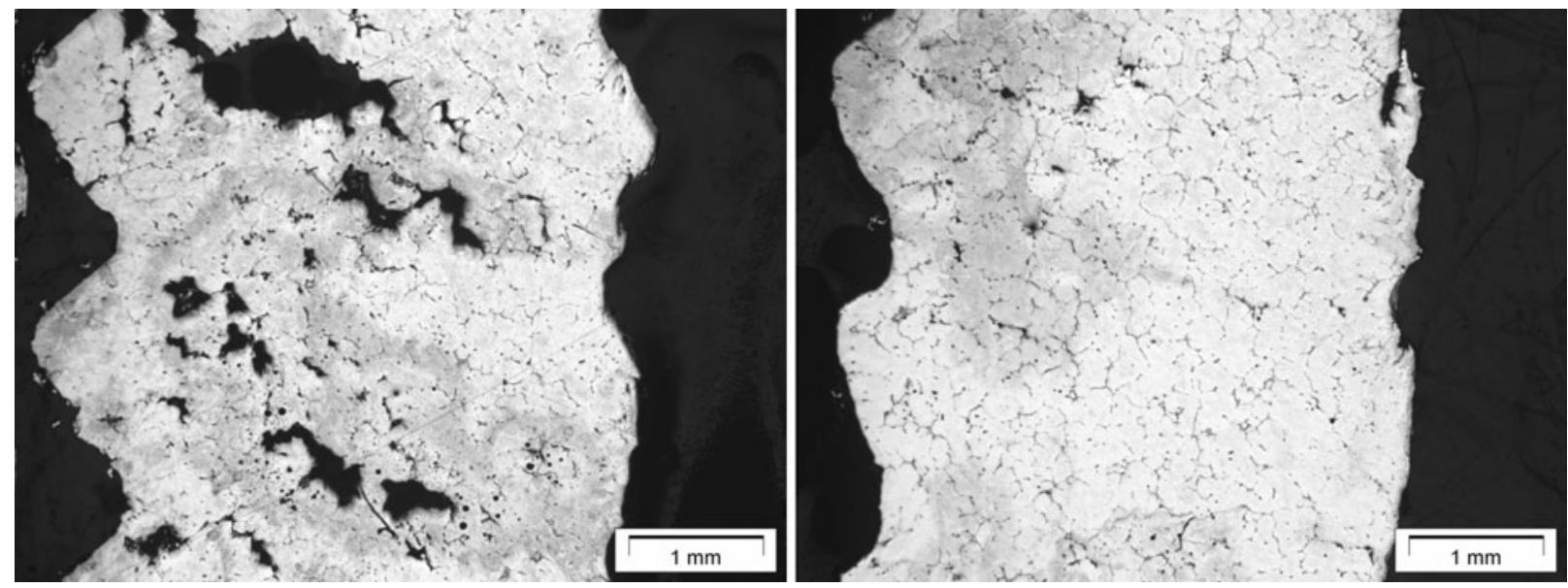

\section{B. Behavior Under Nonisothermal Conditions}

The isothermal shear test allows the behavior of the solidifying alloys to be investigated in more realistic and more complex conditions than under isothermal conditions. During this test, the alloy is deformed while the solid fraction increases. Stress obviously increases with increasing strain or solid fraction, because more and more solid-solid contacts formed. Based on the previous results obtained in isothermal conditions, it was expected that the stress increase would be steeper at the solid fraction for coalescence, i.e., at a solid fraction of 0.97 . This is not the case and even a slower increase is observed at a solid fraction of 0.95 to 0.96 , particularly for the higher strain rate. This leads to a larger stress for the lower strain rate at a solid fraction equal to 1 .

The explanation for this surprising behavior can be found by looking at the microstructure of the specimens after deformation (Figure 9). At high strain rate, damage occurred quite extensively, thus leading to a decrease of the shear stress. This damage is linked to the accumulated strain experienced by the specimen before solidification is completed. Indeed, since the durations of the tests are the same and correspond to the time required for the solid fraction to increase from 0.8 to 1 , it should be noticed that the accumulated strain is larger for the higher strain rate (Table II). In particular, in the critical range for which the material is relatively brittle (i.e., for solid fractions between 0.9 and 0.97$),{ }^{[5]}$ the accumulated strain most probably exceeded the strain for cracks to occur.

Table II. Accumulated Strain During Nonisothermal Experiments at Various Strain Rates

\begin{tabular}{lcc}
\hline & $\begin{array}{c}\text { Accumulated } \\
F_{\mathrm{s}}\end{array}$ & $\begin{array}{c}\text { Strain (Pct) at } 0.001 \mathrm{~s}^{-1} \\
\text { Strain (Pct) at } 0.0005 \mathrm{~s}^{-1}\end{array}$ \\
\hline 0.9 & 4.7 & 2.1 \\
0.97 & 13.6 & 6.8 \\
1 & 25 & 12.8 \\
\hline
\end{tabular}

Fig. 9-Microstructures obtained after non isothermal shear experiments carried out from an initial solid fraction of 0.8 with a cooling rate of $0.3 \mathrm{~K} / \mathrm{s}$ and a strain rate of $(a) 0.001 \mathrm{~s}^{-1}$ and $(b) 0.0005 \mathrm{~s}^{-1}$ - Flick's etch. 
Conversely to the high strain rate experiments, damage did not occur at low strain rate, as shown in Figure 9. In this case, the accumulated strain was probably too small to induce crack formation. Another explanation could be that forming cracks were healed by liquid flow in the critical solid fraction range.

\section{Modeling of the Shear Behavior Under Both Thermal Conditions}

Since shear experiments under isothermal conditions showed that the deformation of the solid controls the deformation of the mush in the solid fraction range $(0.9$ to 1 ), it is possible to model the shear behavior of the alloy by considering the model developed by Ludwig et $a l^{\left[{ }^{[7]}\right.}$ They treated the solidifying alloy as a viscoplastic porous medium saturated with liquid. The behavior is controlled by the solid phase, and the influence of the liquid phase is taken into account in two ways. The first one corresponds to the softening effect of the liquid simply considered as pores. The second one is linked to the variation of the cohesion of the solid phase due to the presence of the liquid as films that wet grain boundaries.

More details can be found in Reference 17 about the model. The plastic strain rate tensor of the solid phase is then defined by the equation

$$
\begin{aligned}
\underline{\underline{\dot{\varepsilon}_{\mathrm{S}}}}= & \frac{K}{C^{n}} \cdot \exp \left(-\frac{Q}{\mathrm{R} T}\right) \cdot\left\{-\frac{A_{2}}{3} \cdot \overline{P_{\mathrm{S}}} \cdot \underline{\underline{1}}+\frac{3}{2} \cdot A_{3} \cdot \underline{\underline{S_{\mathrm{S}}}}\right\} \\
& \cdot\left\{A_{2} \cdot{\overline{P_{\mathrm{S}}}}^{2}+A_{3} \cdot{\overline{\sigma_{\mathrm{S}}}}^{2}\right\}^{\frac{n-1}{2}}
\end{aligned}
$$

where $C$ is the cohesion of the solid; 1 the unit tensor; $\overline{P_{\mathrm{S}}}$ the effective pressure on the solid skeleton; $\overline{\sigma_{\mathrm{S}}}$ the Von Mises stress; $S_{\mathrm{S}}$ the solid-phase deviatoric effective stress tensor; $A_{2}$ and $A_{3}$ the solid fraction functions; $n, K$, and $Q$ the material parameters in the fully solid state; and $T$ the temperature.

In the case of a shear stress state, the effective pressure $\overline{P_{\mathrm{S}}}$ is nil. Moreover, since the equivalent macroscopic plastic strain rate of the solid skeleton (i.e., the scalar measure) is defined by $\dot{\varepsilon}_{e}=\sqrt{\frac{2}{3}} \underline{\underline{\dot{\varepsilon}_{\mathrm{S}}}}: \underline{\underline{\dot{\varepsilon}_{\mathrm{S}}}}$, Eq. [1] becomes

$$
\dot{\varepsilon}_{e}=\frac{K}{C^{n}} \cdot \exp \left(-\frac{Q}{\mathrm{R} T}\right) \cdot A_{3}^{\frac{n+1}{2}} \cdot{\overline{\sigma_{\mathrm{S}}}}^{n}
$$

The material parameters $(n, K$, and $Q)$ are determined by carrying out compressive tests on the fully solid alloy at high temperatures and at various strain rates. The tested temperatures were $723 \mathrm{~K}, 773 \mathrm{~K}, 803 \mathrm{~K}$, and $823 \mathrm{~K}\left(450{ }^{\circ} \mathrm{C}, 500{ }^{\circ} \mathrm{C}, 530{ }^{\circ} \mathrm{C}\right.$, and $\left.550{ }^{\circ} \mathrm{C}\right)$. The applied strain rates were $1 \times 10^{-4}, 2.5 \times 10^{-4}$, $1 \times 10^{-3}, 2.5 \times 10^{-3}$, and $6 \times 10^{-3} \mathrm{~s}^{-1}$. The analysis

Table III. Material Parameters Used in the Rheological Law Given by Equation [2]

\begin{tabular}{lcc}
\hline$n$ & $Q(\mathrm{~kJ} / \mathrm{mol})$ & $\mathrm{K}\left(\mathrm{s}^{-1} \cdot \mathrm{MPa} \mathrm{m}^{1 / 2}\right)$ \\
\hline 12 & 347 & $4.6 \times 10^{5}$ \\
\hline
\end{tabular}

of the stress-strain curves (not shown in this article) leads to the material parameters given in Table III by considering that the rheology of the fully solid phase is described by a simple creep law: $\dot{\varepsilon}=K \cdot \sigma^{n} \cdot \exp \left(-\frac{Q}{R T}\right)$. The values of $n$ and $Q$ are quite high compared with the usually reported values; generally, solid alloys exhibit a stress exponent of about 5 , and the activation energy is close to that for the diffusion of $\mathrm{Al}$ in an $\mathrm{Al}$ matrix, i.e., about $130 \mathrm{~kJ} / \mathrm{mol}^{[21,22]}$ These high values indicate that the rheological behavior of this alloy at high temperature can not be described by this type of equation but rather by an equation with a hyperbolic sine. ${ }^{[23-27]}$ For some alloys, it is possible to reach a field of stresses and strain rates for which a transition occurs between a power-law regime described by an exponential law and a power-law breakdown regime described by a hyperbolic sine law: stress increases faster than what can be predicted by an exponential law. This equation will be used nevertheless for the behavior of the solid phase with the values of $n$ and $Q$ given in Table III.

The function $A_{3}$ represents the softening effect of the liquid in the form of pockets. The same equation as that used by Ludwig et al. ${ }^{[7]}$ is applied:

$$
A_{3}=\left[1+\frac{2}{3} \cdot\left(1-F_{\mathrm{s}}\right)\right] \cdot F_{\mathrm{s}}^{\frac{-2 \cdot \mathrm{n}}{\mathrm{n}+1}}
$$

where $F_{\mathrm{s}}$ is the solid fraction.

The cohesion parameter $C$ is between 0 and 1: $C$ is equal to 0 when the grains are fully wetted by the liquid, and $C$ is equal to 1 when grains coalesce and only liquid pockets remain. Ludwig et al.$^{[7]}$ considered that $C$ evolves with time owing to the rearrangement of solid grains: $C$ increases thanks to the formation of contacts between solid grains and decreases by contact failure. The evolution equation of $C$ is

$$
\frac{d C}{d t}=\alpha \cdot\left(1-\frac{C}{C^{*}}\right) \dot{\varepsilon}_{e}
$$

where $\alpha$ is a function governing the variation of $C$ at small strains and $C^{*}$ is the saturation value of $C$ at large strains.

By considering that $\alpha$ and $C^{*}$ are constant during shear experiments (i.e., time independent), Eq. [4] can be integrated:

$$
C=C^{*}+\left(C_{0}-C^{*}\right) \cdot \exp \left(-\frac{\alpha}{C^{*}} \cdot \varepsilon_{e}\right)
$$

where $C_{0}$ is the initial cohesion of the solid, which depends on the solid fraction. $C_{0}$ is chosen arbitrarily, but three conditions are imposed: (1) $C_{0}$ is equal to 0 when the solid fraction is about 0.6 , which corresponds to the generally accepted value of the coherency solid fraction for globular aluminum alloys; (2) $C_{0}$ is equal to 1 when the alloy is fully solid; and (3) the variation of the initial cohesion with increasing solid fraction exhibits the same shape as the variation of stress (shown in Figure 5): a slow increase at low solid fraction followed by a sharp acceleration when coalescence occurs. These conditions are satisfied by 

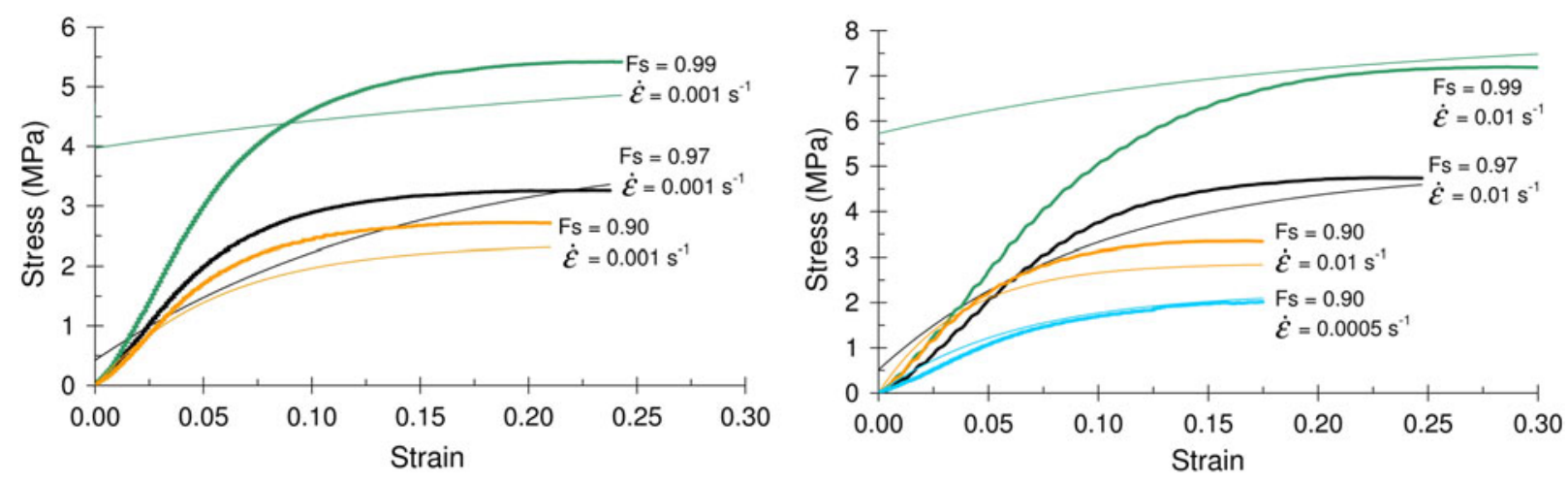

Fig. 10 - Comparison between the experimental variation of stress (thick lines) with strain and the modeled variation (thin lines) for isothermal shear tests carried out at various solid fractions $\left(F_{\mathrm{s}}\right)$ and strain rates $(\dot{\varepsilon})$.

using the following equation, which relates $C_{0}$ to the solid fraction $F_{\mathrm{s}}$ :

$$
C_{0}=\exp \left(a \cdot F_{\mathrm{s}}-b\right)
$$

with $a=b=107.3$.

The behavior of the semisolid alloy under a shear stress state, therefore, is defined via Eqs. [2], [3], [5], and [6]. These equations will be used to model the behavior under isothermal and nonisothermal conditions. However, for each condition, appropriate expressions of the $\alpha$ and $C^{*}$ have to be determined.

\section{Modeling of the shear behavior under} isothermal conditions

By fitting the stress-strain curves (shown in Figure 4), the expressions of $\alpha$ and $C^{*}$ can be determined:

$$
C^{*}=1-\left(1-F_{\mathrm{s}}\right)^{p} \text { and } \alpha=\alpha_{1} \cdot \dot{\varepsilon}_{e}^{\alpha_{2}} \cdot\left(1-F_{\mathrm{s}}\right)^{\alpha_{3}}
$$

with $p=0.14, \lambda=1 \cdot 10^{-3}, \alpha_{1}=30, \alpha_{2}=0.17$, and $\alpha_{3}=0.36$.

A comparison between the experimental curves and the curves given by the model (application of Eqs. [2], [3], [5], [6], and [7]) is shown in Figure 10. The thin lines are obtained with the model.

The agreement is relatively good, particularly at large strains, which is due to the appropriate choice of the saturation value $C^{*}$ of the cohesion. The differences at small strains (close to 0 ) are mainly due to the initial cohesion $C_{0}$. During experiments for which the load is recorded with a load cell set initially at zero, the stress is obviously equal to zero at the beginning of the test whatever the solid fraction and, thus, whatever the initial cohesion. In the model, a given initial solid fraction corresponds to an initial cohesion and, thus, to an initial stress, which is more or less large depending on this solid fraction. Therefore, the model is not able to take into account the initial transition in the loading curve.

\section{Modeling of the shear behavior under} nonisothermal conditions

During these tests, the solid fraction increases so that it is necessary to relate the macroscopic strain $\varepsilon_{e}$ in Eq. [5] to the solid fraction.
The macroscopic strain can be written as

$$
\varepsilon_{e}=\frac{\dot{\varepsilon}_{e}}{\dot{T}}\left(T_{0}-T\right)
$$

where $\dot{T}$ is the cooling rate and $T_{0}$ is the temperature at which the experiment starts (i.e., the initial temperature).

The term $T$ is related to the solid fraction by the curve given in Figure 3. A simple empirical expression for this curve can be found, leading to the following expression for the macroscopic strain:

$$
\varepsilon_{e}=\frac{\dot{\varepsilon}_{e}}{\dot{T}}\left(\frac{F_{\mathrm{s} 0}-f_{1}}{f_{3}-f_{2} \cdot F_{\mathrm{s} 0}}-\frac{F_{\mathrm{s}}-f_{1}}{f_{3}-f_{2} \cdot F_{\mathrm{s}}}\right)
$$

where $F_{\mathrm{s} 0}$ the initial solid fraction, $f_{1}=1.096, f_{2}=$ $-1.075 \cdot 10^{-3}$, and $f_{3}=-1.189 \cdot 10^{-3}$.

By inserting Eq. [9] into Eq. [5] and by assuming that the saturation value of the cohesion is equal to 1 (since the test is carried out until the solid state is reached), the expression of the cohesion is given by

$$
C=1+\left(C_{0}-1\right) \exp \left(-\alpha \cdot \frac{\dot{\varepsilon}}{\dot{T}} \cdot\left(\frac{F_{\mathrm{s} 0}-f_{1}}{f_{3}-f_{2} F_{\mathrm{s} 0}}-\frac{F_{\mathrm{s}}-f_{1}}{f_{3}-f_{2} F_{\mathrm{s}}}\right)\right)
$$

As for isothermal tests, the values of $\alpha$ have to be determined from the stress-solid fraction curves (shown in Figure 8). However, since there are not enough data to define an expression for $\alpha$, an average value has been used, i.e., 4.4.

The comparison between the experimental results (thick lines) and those obtained with the model (thin lines) is shown in Figure 11. The agreement is relatively good.

At the beginning of the test (when the solid fraction is equal to the initial solid fraction), there is almost no difference between the initial stress given by the model and the experimental stress contrary to isothermal conditions. This is due to the fact that the tests were performed at an initial solid fraction of 0.8 for which the initial cohesion is still very small, leading to a very small (close to 0) initial stress. 


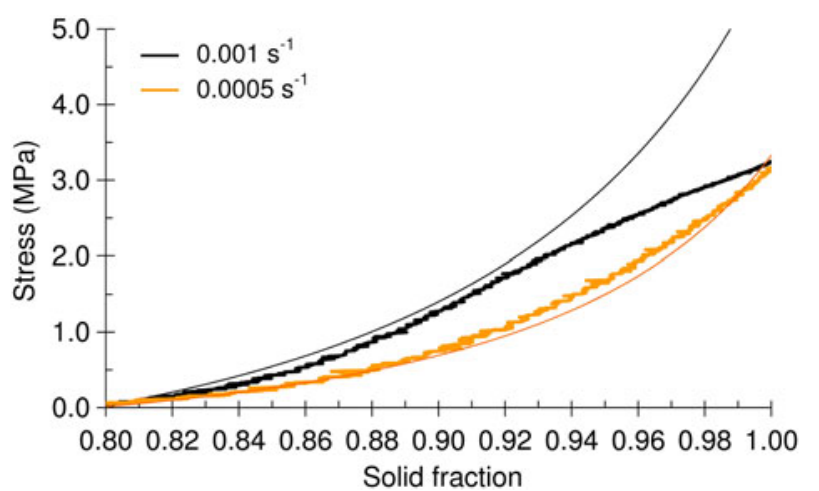

Fig. 11-Comparison between the experimental variation of stress (thick lines) with solid fraction and the modeled variation (thin lines) for nonisothermal shear tests carried out at various strain rates from an initial solid fraction of 0.8 at a cooling rate of $0.3 \mathrm{~K} / \mathrm{s}$.

At high solid fractions and for the highest strain rate, the curve given by the model moves away from the experimental curve. This disagreement can be explained by the occurrence of damage (Figure 9) in the material within the critical solid fraction range (0.90 to 0.97$)$. This damage is not considered in the model, since it describes the behavior of the sound mushy alloy.

\section{CONCLUSIONS}

The shear behavior in the semisolid state of a 6061 aluminum alloy was investigated by performing translation shear tests in which the material is deformed in between two concentric cylinders. The tests were carried out during solidification of the alloy at large solid fractions under either isothermal or nonisothermal conditions.

In isothermal conditions, the shear stress strongly depends on the solid fraction with a transition at the solid fraction for coalescence of the dendrites. The behavior of the material is viscoplastic with values of the strain-rate-sensitivity parameter typical of the deformation of the solid at high temperature. These results are in agreement with those obtained during tensile tests, ${ }^{[5]}$ which shows that the alloy undergoes the same behavior whatever the stress state.

Under nonisothermal conditions, stress obviously increases with increasing solid fraction; however, at a relatively high strain rate, deformation is associated with crack formation, which develops owing to the decrease of the liquid fraction in the latest stages of solidification. The amount of accumulated strain during solidification appears to be an important parameter for crack formation, in agreement with some strain-based criteria. ${ }^{[1,8]}$

Modeling of the shear behavior by using the model developed by Ludwig et al. ${ }^{[7]}$ leads to fair predictions whatever the thermal conditions. The use of a cohesion parameter of the solid phase allows taking into account the effect of solid fraction, strain rate, and thermal condition on the behavior of the semisolid state.

\section{ACKNOWLEDGMENTS}

One of the authors (EG) is grateful to CNRS (French National Center for Scientific Research) and AREVA for financial support through a scholarship. The authors thank Cédric Gasquères, ALCAN CRV, for providing the ProPhase calculations.

\section{REFERENCES}

1. D.G. Eskin, Suyitno, and L. Katgerman: Progr. Mater. Sci., 2004, vol. 49, pp. 629-711.

2. A.K. Dahle and L. Arnberg: JOM, 1996, vol. 48, pp. 34-37.

3. M. Rappaz, J.M. Drezet, and M. Gremaud: Metall. Mater. Trans. A, 1999, vol. 30A, pp. 449-55.

4. E. Giraud, M. Suéry, J. Adrien, E. Maire, and M. Coret: in Hot Cracking Phenomena in Welds III, 1st ed., T. Böllinghaus, C. Lippold, and C.E. Cross, eds., Springer, 2011, pp. 87-101.

5. E. Giraud, M. Suéry, and M. Coret: Metall. Mater. Trans. A, 2010, vol. 41A, pp. 2257-68.

6. D. Fabrègue, A. Deschamps, M. Suéry, and W.J. Poole: Metall. Mater. Trans. A, 2006, vol. 37A, pp. 1459-67.

7. O. Ludwig, J.M. Drezet, C.L. Martin, and M. Suéry: Metall. Mater. Trans. A, 2005, vol. 36A, pp. 1525-35.

8. B. Magnin, L. Maenner, L. Katgerman, and S. Engler: Mater. Sci. Forum, 1996, vols. 217-222, pp. 1209-14.

9. W.M. van Haaften, W.H. Kool, and L. Katgerman: Mater. Sci. Forum, 2000, vols. 331-337, pp. 265-70.

10. C.E. Cross: in Hot Cracking Phenomena in Welds, T. Bollinghaus and H. Herold, eds., 2005, pp. 3-18.

11. S. Terzi, L. Salvo, M. Suéry, N. Limodin, J. Adrien, E. Maire, Y. Pannier, M. Bornert, D. Bernard, M. Felberbaum, M. Rappaz, and E. Boller: Scripta Mater., 2009, vol. 61, pp. 449-52.

12. D. Fabrègue, A. Deschamps, M. Suéry, and W.J. Poole: Mater. Sci. Forum, 2006, vols. 519-521, pp. 1877-82.

13. M.G. Chu and A. Granger: Mater. Sci. Forum, 1996, vols. 217 222, pp. $1505-10$.

14. A.K. Dahle, S. Instone, and T. Sumitomo: Metall. Mater. Trans. A, 2003, vol. 34A, pp. $105-13$.

15. M.R. Twite, J.A. Spittle, and S.G.R. Brown: Int. J. Form. Processes, 2004, vol. 7, pp. 233-60.

16. E. Giraud, M. Suéry, and M. Coret: Proc. 12th Int. Conf. on Aluminum Alloys, The Japan Institute of Light Metals, Tokyo, 2010, pp. 167-72.

17. O. Ludwig, C.L. Martin, and M. Suéry: Mater. Sci. Forum, 2002, vols. $396-402$, pp. $265-70$

18. O. Ludwig, J.M. Drezet, P. Ménésès, C.L. Martin, and M. Suéry: Mater. Sci. Eng. A, 2005, vols. 413-414, pp. 174-79.

19. C.L. Martin, S.B. Brown, D. Favier, and M. Suéry: Acta Mater., 2006, vol. 54, pp. 5209-20.

20. A.K. Dahle and L. Arnberg: Acta Mater., 1997, vol. 45, pp. 54759.

21. B.S. Bokstein: Mater. Sci. Forum, 1996, vols. 217-222, pp. 685-88.

22. X. Doré, H. Combeau, and M. Rappaz: Acta Mater., 2000, vol. 48 , pp. 3951-62.

23. F.R.N. Nabarro: Acta Mater., 2006, vol. 54, pp. 263-95.

24. H.J. McQueen, E. Fry, and J. Belling: JMEPEG, 2001, vol. 10, pp. $179-83$.

25. M.S. Soliman: J. Mater. Sci., 1987, vol. 22, pp. 3529-32.

26. S. Spigarelli, E. Evangelista, and H.J. McQueen: Scripta Mater., 2003, vol. 49, pp. 179-83.

27. E.A. El-Danaf, A.A. Almajid, and M.S. Soliman: JMEPEG, 2008, vol. 17 , pp. $572-79$. 